\title{
Stiction Compensation in Agonist-Antagonist Variable Stiffness Actuators
}

\author{
Luca Fiorio Francesco Romano Alberto Parmiggiani Giulio Sandini and Francesco Nori \\ Department of Robotics Brain and Cognitive Sciences \\ Istituto Italiano di Tecnologia, 16163 Genova, Italy \\ Email: name.surname@iit.it
}

\begin{abstract}
In the last decade new actuator designs have been presented trying to introduce at mechanical level the advantages of compliance. Ranging from serial elastic actuators to different designs of variable stiffness actuators, various prototypes have been proposed and implemented on robots, thus allowing performance of novel and challenging tasks. Nevertheless some of these new devices often are affected by the drawbacks related to friction. In particular, static friction due to its discontinuous nature, can produce undesired behaviors that are rather difficult to compensate.

In this paper we present a novel kind of passive variable stiffness actuator based on agonist-antagonist configuration. The specific design we adopted improves the capability of the system in mechanically compensating the external disturbances, but on the other hand intensifies the effect of stiction during the cocontraction of the agonist and antagonist side of the actuator. The consequence is the appearance of a set of neutral equilibrium configurations of the output joint that we named "dead-band". This issue is tackled analytically investigating the propagation and the distribution of the stiction components within the whole system. The result is a condition over the spring potential energies that is exploited to properly design the new non-linear springs. Eventually experimental tests are conducted on the real actuator, showing the effectiveness of our analytical approach.
\end{abstract}

\section{INTRODUCTION}

Over the last decades robot technology has experienced remarkable progresses. Rise in processing power of electronic micro-controllers and computers has lead to noteworthy improvements in planning and robot control. From a mechanical standpoint, advances were closely tied to the development of new actuation systems. Series elastic actuators (SEA) [1] nowadays represent an alternative to classic rigid actuators composed by electric motors and gears. More recently, several variable stiffness actuators (VSA) [2] have also been introduced to overcome some of the SEA limitations, which arise from the constant value of actuator stiffness. In spite of greater advantages in terms of reliability, safety and energy storage [3], these novel generations of systems are affected by new issues that are mainly related to their increased mechanical complexity. In particular, with reference to agonist-antagonist VSA, friction is often identified as one of the most evident and most adverse drawback. All antagonistic actuators are based on the basic idea of co-contracting both agonist and antagonist motor sides of the system, to increase joint stiffness. As a consequence, internal forces increase thus augmenting frictions, and in particular stiction components. Eventually, the performance of the actuator are highly affected by the nonlinearities introduced by stiction and its relative discontinuities are often difficult to compensate. This decay in performance is, together with inertia issues, a limit for controlling the actuator mechanical bandwidth while performing applications such as torque control or dynamic tasks involving human or environment interaction. Different approaches have been proposed to reduce most evident and undesirable drawbacks. Among these there are classical integrator action and disturbance observer [4], adaptive controllers [5], sliding mode control [6] or modelbased friction compensation [7]. All of these approaches have the advantage of being fast and accurate, but most of them reduce the benefit of inherent passive compliance to a certain extent. Controllers indeed introduce an additional compliance which acts upon the series, variable or fixed, elasticity and that could be difficult to tune.

In this paper we focus our analysis on a new actuator [8], that can be classified in the group of unconventional agonistantagonist variable stiffness actuators, which comprises, for example, the quasi-antagonistic design [9], the bidirectional design [10] and the cross-coupled design [11]. Due to the particular structure of the system, the actuator output joint should posses a unique equilibrium position [12], but the presence of stiction gives rise to a set of indifferent equilibrium configuration. This range of equilibrium position, named "dead-band", rapidly increases together with actuator co-contraction, i.e. simultaneous activation of the agonist and antagonist actuators. Starting from the observation that both friction and spring restoring forces can be represented as a function of the actuator internal tension, we explore the possibility of mechanically compensate the stiction adverse effects by exploiting the actuator elastic elements. Through the analytical representation of the correlations of the system internal states, we derive analytical conditions to ensure that during co-contraction the increase of the restoring forces is "faster" than the increase of the friction forces. We exploit this representation to formulate differential conditions on the spring potential energy to guarantee that co-contraction reduces the effect of friction on the joint equilibrium position. The design of an optimized set of springs, respecting these conditions, led to the construction of a new version of our actuator. This new model shows that for increasing levels of co-contraction the effect of stiction, and thus the dead-band effect, decreases. 
The main contribution of this work resides in a complete and unique technique to mathematically model the relationship between stiction and spring restoring forces, deduce analytical conditions over the spring potential energies and design new springs to improve the actuator performances in terms of static dead-band. Furthermore our analysis does not make any restrictive assumptions on the spring characteristic, making this procedure as general as possible.

The rest of the paper is organized as follows. Section II gives an overview of the fundamental principles that guided the actuator design. Section III presents the "case study" prototype focusing on its main features and introducing the analytical description of the sensitivity of the internal states. In Section IV we introduce the issue caused by the stiction that we aim to solve by identifying the main friction source and by providing the mathematical representation of the dead-band. In Section $\mathrm{V}$ we present the main theoretical analysis focused on understanding which conditions the non-linear springs should satisfy to reduce the effects of stiction, and providing the relative closed form solution. Finally, Section VI is dedicated to the finding of an optimum design of the non-linear springs, which have been tested on the real system for the validation of our theoretical analysis.

\section{BACKGROUND}

The system considered in this paper is a novel agonistantagonist VSA that has been designed to tackle highly dynamical tasks. This new design principle arises from the requirement to have new robotic systems able to operate reliably in unstructured environments, where robots with rigid structure are becoming a limitation for envisioned tasks or applications such as in human-robot interaction [13]. To face this problem different approaches have been proposed from impedance control of stiff actuators like the DLR-LWR [14] to several mechanical architectures to adjust the transmission rigidity [15] and improve safety interaction [16]. Nevertheless some drawbacks are starting to emerge because relying on feedback in artificial agents (such as humanoid robots) might not be a practical strategy specifically considering the growing amount of sensors (e.g., distributed force/torque sensors [17], whole-body distributed tactile sensors [18], gyros and accelerometers [19]) which are currently available and have to be acquired and centrally processed to perform complex actions. The design behind our actuator has been inspired by investigating the muscle co-activation strategy that humans exploit to cope with sensorimotor delays and noise in presence of instabilities [20]. What results from these studies is that stiffness regulation is indeed an essential movement feature that in biological systems is not achieved with active feedback loops.

\section{A. passive noise rejection}

In a recent work we considered a broad class of pVSA and we computed the associated passive disturbance rejection [21]. In Fig. 1 we depict from a mechanical standpoint some of the interaction that can occur between a motor $\vartheta$, a joint $q$ and

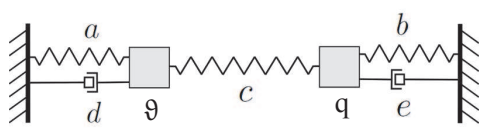

Fig. 1. Schematic representation of an actuation system. The elastic and damping elements $a$ and $b, c$ and $d$ correspond to the linearization of the environmental interactions with the joint $q$ and the motor $\vartheta$ respectively.

the environment. For example the key component of a typical pVSA is an elastic element of variable stiffness in between the motor and the joint, this elastic element is represented by the spring $c$. We study the effect of disturbances entering the system focusing in the passive disturbance rejection, i.e. no feedback has been considered to increase disturbance rejection. Disturbance is represented by stochastic variables acting as forces on the motor and on the joint (white noise). Computations, not reported here given the complexity of the analytical expression [21], show that the passive disturbance rejection monotonically increases with the stiffness of the elastic element in between the motor and the joint (represented by the parameter $c$ ). However, the values of $a, b, d$ and $e$ are also essential to guarantee a certain level of passive disturbance rejection. The spring elements connecting the motor and the joint to the environment, $a$ and $b$ respectively, are typically not present in pVSA designs with rotary motors. Nevertheless, they play a crucial role in determining the overall system passive disturbance rejection. In practice when $a=b=0$ the system is free-floating with respect to the environment and noise can drive the system arbitrarily far from the initial configuration. In a sense passive noise rejection is increased by augmenting the stiffness of the path which connect the joint to the ground. Therefore, we concentrate on actuators like the ones in Fig. 1 not having $a$ and $b$ simultaneously zero. We name these actuators "passive noise rejecting VSA" (pnrVSA).

A nice example of actuators possessing the aforementioned features is represented by biological muscles. In particular the main properties of the biological muscles have been reported by [22] using the model represented in the left part of Fig 2 It can be proven (see [23] pag.23) that its mechanical model is equivalent to the one shown in the right part of Fig. 2 and therefore the overall muscle force can be written as:

$$
F=F_{S E}\left(K_{S E}, l_{2}\right)=F_{P E}\left(K_{P E}, l_{1}\right)+\vartheta\left(L_{j}, f(t)\right),
$$

where $K_{S E}$ is the series elastic element ( $c$ in Fig. 1), $K_{P E}$ is the parallel elastic element ( $a$ in Fig. 1) which in series with $K_{S E}$ account for the passive tension properties of the muscle and $\vartheta$ is the active force generated by the contractile element depending on the muscle history activation $f(t)$ and the overall length $L_{j}$.

During the actuator design we concentrate on two main characteristics of the mechanical muscle model: the possibility of finding a closed path that connects the frame to the actuator endpoint and the capability of varying the stiffness independently from length. Thus our goal has been to design a mechanism composed by an elastic element in series with a 

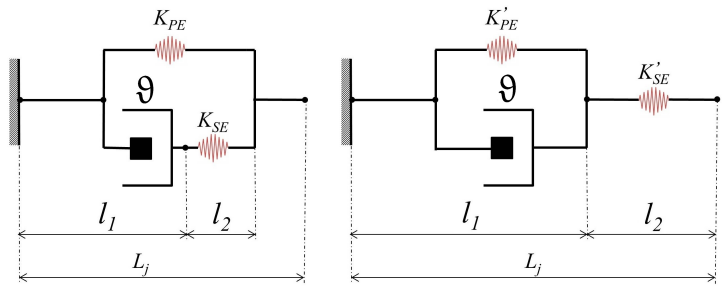

Fig. 2. Hill muscle model (right hand side) and an equivalent model (left hand side). Muscle regulation, as suggested by [24], is done controlling the system as a non-linear spring with adjustable resting length. The actuator $\vartheta$ is represented as a contractile element.

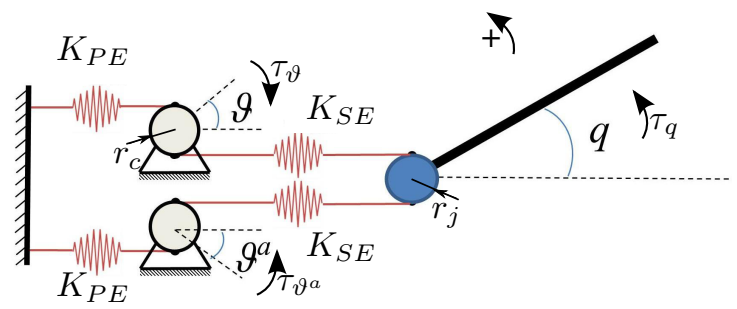

Fig. 3. Prototype schema: output joint $q$ is controlled by means of internal actuation torques $\tau_{\vartheta}$ and $\tau_{\vartheta} a$, while $\vartheta$ and $\vartheta^{a}$ represent capstan angular position. $\tau_{q}$ is an external torque acting on the output joint.

structure composed by a contractile element in parallel with a second elastic element 1 . As shown in Fig. 3 this idea has been exploited to build an agonist-antagonist actuator which resembles the human muscles arrangement: all springs connecting the joint to the motors and the motors to the ground are non-linear (as indicated by the sketch with coils of variable width).

\section{PROTOTYPE DESCRIPTION}

We now propose an overview of the actuator mechanical design focusing on the nonlinear spring selection and design. The principle sketched in Fig. 1 1 has been implemented with rotational motors which are more commonly used in the foreseen applications. The design shown in Fig. 3 is obtained by attaching two nonlinear springs on the opposite side of the motor capstans: the first, named $K_{P E}$, realizes the connection to the ground and works in parallel with respect to the contractile element $\vartheta$ (spring elongation equals reel displacement); the second, named $K_{S E}$, behaves as series elastic element. Intuitively, a clockwise rotation of the motor $\vartheta$ coupled with a counterclockwise rotation of $\vartheta^{a}$ stretches all springs causing no movement of the joint $q$. If springs are designed in such a way that stiffness increases with stretch, the overall path connecting the joint to the ground is stiffened up, resulting in increased noise rejection. A complete analysis and characterization of these intuitive control laws can be found in [12]. Furthermore we can define the following quantities:

- $r_{j}$ is the radius of the joint pulley;

- $r_{c}$ is the capstan radius;

${ }^{1}$ With reference to Fig. 1] we can identify this mechanical structure considering the the components $a, \vartheta$ and $c$.
- $\vartheta$ is the agonist capstan angular position;

- $\vartheta^{a}$ is the antagonist capstan angular position;

- $q$ is the joint angular position.

As a convention for positive rotations of all angles $\left(\vartheta, \vartheta^{a}\right.$ and $q$ ), we will assume positive angles for counterclockwise rotations. Since the device has been designed to work in a co-contraction configuration we can simplify our analysis by assuming $\vartheta$ always negative $(\vartheta<0)$ and $\vartheta^{a}$ always positive $\left(\vartheta^{a}>0\right)$ corresponding to the black arrows in Fig. 3 .

The nonlinear spring design has been optimized and customized in order to have light and compact solutions for both the parallel elastic element $K_{P E}$ and the series elastic element $K_{S E}$. The change in stiffness is achieved through a cam varying radius, specialized into two different custom solutions. Regarding the $K_{S E}$ spring we rely on a similar procedure to the one reported in [25], connecting a non-circular spool in parallel to a linear torsional spring, as shown in the left hand side of Fig. 4. In designing the parallel elastic element $K_{P E}$ we had to consider some constraints related to the wide range of motion of the output joint. Therefore we adopted a different design, which relies on a three-dimensional noncircular profile (right hand side of Fig. (4) connected to a linear compression spring. In Fig. 5 is shown the CAD model of the complete actuator for which more details can be found in [8].
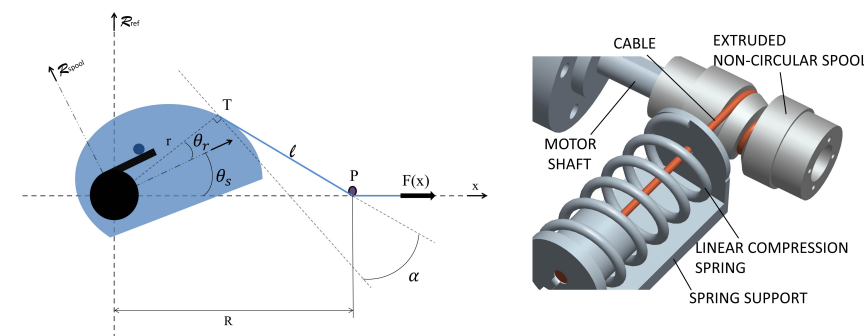

Fig. 4. The nonlinear springs. On the left side the scheme that represents the principle used to derive the $K_{S E}$ elastic element which can be seen as a nonlinear extensional spring. While on the right side a schematic representation of the $K_{P E}$ elastic element, which can be considered as a non-linear torsional spring.

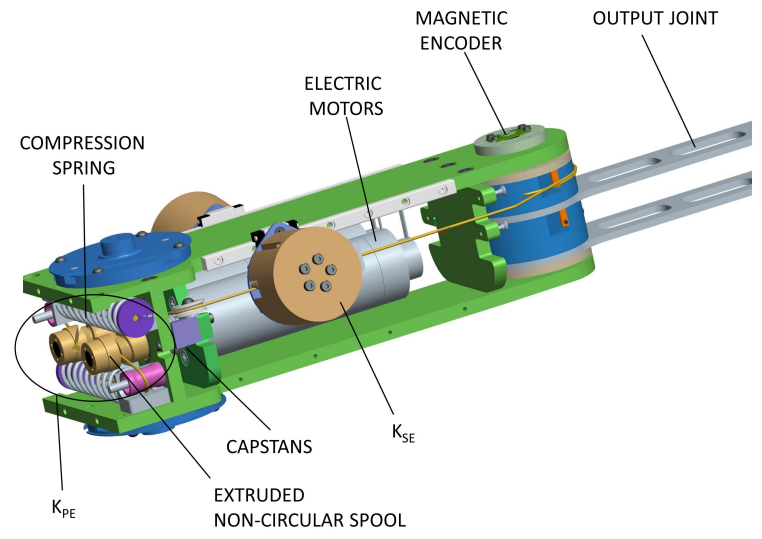

Fig. 5. Overview of the CAD model of the pnrVSA. 


\section{A. Sensitivity matrix derivation}

To analyze the system behavior we need to introduce a mathematical description of the relationship between the actuator internal states. For this purpose with reference to the diagram in Fig. 3 we introduce the potential energies of the spring as $U_{1}$ and $U_{4}$ respectively for the agonist and antagonist $K_{P E}$ elastic elements and as $U_{2}$ and $U_{3}$ for the $K_{S E}$ elastic elements. Moreover assuming equilibrium configurations $\left(q_{e q}, \vartheta_{e q}, \vartheta_{e q}^{a}\right)$ with constant torques $\left(\tau_{q}=\bar{\tau}_{q}\right.$, $\tau_{\vartheta}=\bar{\tau}_{\vartheta}$ and $\left.\tau_{\vartheta^{a}}=\bar{\tau}_{\vartheta^{a}}\right)$ we can write the following equations:

$$
\left\{\begin{array}{l}
-U_{1}^{\prime}\left(\vartheta_{e q}\right)-U_{2}^{\prime}\left(\vartheta_{e q}+q_{e q}\right)=\bar{\tau}_{\vartheta} \\
U_{4}^{\prime}\left(\vartheta_{e q}^{a}\right)+U_{3}^{\prime}\left(-q_{e q}+\vartheta_{e q}^{a}\right)=\bar{\tau}_{\vartheta a} \\
-U_{2}^{\prime}\left(\vartheta_{e q}+q_{e q}\right)+U_{3}^{\prime}\left(-q_{e q}+\vartheta_{e q}^{a}\right)=\bar{\tau}_{q}
\end{array},\right.
$$

where $U_{i}^{\prime}(x)$ is the first derivative of the potential energy with respect to $x$. These equations define a functional which can be exploited to define the relationship between the internal torques $\tau=\left[\hat{\tau}_{\vartheta}, \hat{\tau}_{\vartheta^{a}}, \hat{\tau}_{q}\right]^{T}$ and capstan/joint position $\alpha=$ $\left[\hat{\vartheta}, \hat{\vartheta}^{a}, \hat{q}\right]^{T}$. In particular to simplify the equations we will consider the following variables:

$$
\begin{aligned}
& r_{c} \vartheta=\hat{\vartheta}, r_{j} q=\hat{q}, r_{c} \vartheta^{a}=\hat{\vartheta}^{a}, \\
& \frac{\tau_{\vartheta}}{r_{c}}=\hat{\tau}_{\vartheta}, \frac{\tau_{q}}{r_{j}}=\hat{\tau}_{q}, \frac{\tau_{\vartheta^{a}}}{r_{c}}=\hat{\tau}_{\vartheta^{a}} .
\end{aligned}
$$

and eventually exploiting the implicit function theorem we can compute the Jacobian matrix:

$$
\frac{\partial \alpha}{\partial \tau}=\left[\begin{array}{ccc}
\frac{\partial \hat{\vartheta}}{\partial \hat{\tau}_{\vartheta}} & \frac{\partial \hat{\vartheta}}{\partial \hat{\tau}_{\vartheta}} & \frac{\partial \hat{\vartheta}}{\partial \hat{\tau}_{q}} \\
\frac{\partial \hat{\vartheta}^{a}}{\partial \hat{\tau}_{\vartheta}} & \frac{\partial \hat{\vartheta}^{a}}{\partial \hat{\tau}_{\vartheta}} & \frac{\partial \hat{\vartheta}^{a}}{\partial \hat{\tau}_{q}} \\
\frac{\partial \hat{q}}{\partial \hat{\tau}_{\vartheta}} & \frac{\partial \hat{q}}{\partial \hat{\tau}_{\vartheta} a} & \frac{\partial \hat{q}}{\partial \hat{\tau}_{q}}
\end{array}\right] .
$$

The analytical expression of $\partial \alpha / \partial \tau$ will play a crucial role in the next section during the modeling of the effects of stiction on the joint $q$. In particular, we will focus on the quantities referred to the sensitivity of the output joint to the internal actuation torques $\frac{\partial \hat{q}}{\partial \hat{\tau}_{\vartheta}}$ and $\frac{\partial \hat{q}}{\partial \hat{\tau}_{\vartheta}^{a}}$, aiming at characterizing how the equilibrium configuration for $q$ is affected by static friction acting on $\vartheta$ and $\vartheta^{a}$.

\section{Stiction EfFects}

As mentioned in the previous section, friction plays an important role in determining the performance of our system. Due to the particular spring and cable configuration that has been chosen to design the actuator, for a given set of internal $\left(\tau_{\vartheta}, \tau_{\vartheta^{a}}\right)$ and external $\left(\tau_{q}\right)$ torques the output joint $q$ should posses a unique equilibrium position, solution of Eq. 进 However, as shown in Fig. 6, the overall system behaves differently and presents multiple equilibrium configurations at which spring restoring and stiction forces are perfectly balanced.

\footnotetext{
${ }^{2}$ In [12] we derived trivial conditions over the potential energies of the springs to ensure the uniqueness of the joint equilibrium position. These conditions are the following: monotonically increasing and non-negative stiffness.
}

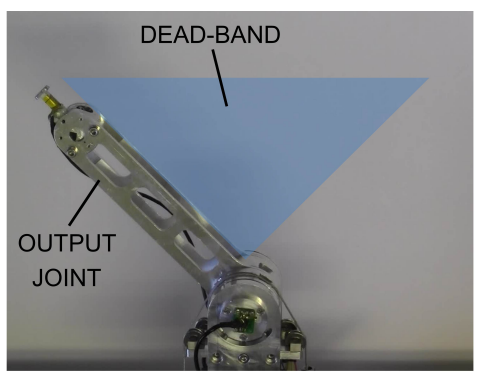

Fig. 6. The picture shows the dead-band that occurs for an internal capstan torque of $1 \mathrm{Nm}$.

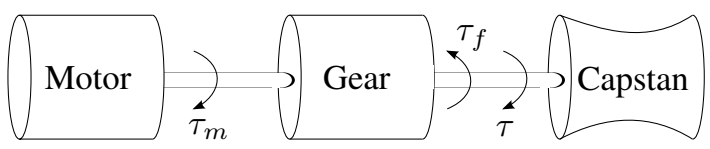

Fig. 7. Motor subsystem: the motor is connected to the capstan by the gear. Friction effects between gear teeth greatly influence the torque transmission from motor to gear and vice-versa.

To identify the main sources of friction we decided to test the actuator by dividing it in two main subsystems: a frame subsystem and a motor subsystem. The frame includes the four non-linear springs, the output joint and all necessary bearings. The motor subsystem, shown in Fig. 7, is composed by the electric motor, the gear and the capstan. This separation has been made knowing that usually the teeth friction in gears is predominant with respect to the other sources. To test the frame subsystem we simply assembled our actuator substituting the two electric motors with their relative gears with two further capstans. These two capstans were connected with a cable to a mass; thus we obtained an ideal constant torque source not affected by gear friction. In this setup we noticed significant improvement of the performances of the system. Already at low level of co-contraction the dead-band effect was hardly noticeable. Further increasing the weight of the masses, thus augmenting the level of co-contraction, rapidly made it disappear. The analysis of the frame subsystem clearly showed that the main source of stiction lies in the motor subsystem.

To explain this behavior we can consider the scheme in Fig. 7 Denoting $\rho$ as the reduction ratio, ideally we should have that $\tilde{\tau}=\rho \tau_{m}$, but in practice the real torque on the load (the internal capstan) $\tau$ can be decreased or increased (depending if considering the subsystem from the motor or the capstan point of view) by the friction torque $\tau_{f}$ which relates to the gear static efficiency.

\section{A. Analytical condition derivation}

To perform our qualitative analysis we assumed that the torque static friction is a function of the torque acting on the load $\tau_{f}=\tau_{f}(\tau)$, with $\tau$ being either $\tau_{\vartheta}$ or $\tau_{\vartheta}$ depending on

\footnotetext{
${ }^{3}$ This assumption derives from the fact that co-activation increases internal forces. Certain friction forces, such as stiction, increase with normal forces and therefore an increased stiction should be expected in response to an increased level of internal forces.
} 
the considered capstan (agonist or antagonist as represented in Fig. 3). The analytical description of the dead-band can be found considering the sensitivity matrix defined in Eq. 2 . We can think of the dead-band as the consequence of an uncertainty that affects the torques that are applied at the capstans, where the amplitude of this uncertainty can be represented considering the stiction torque. Furthermore the total actuator dead-band $\triangle q$ can be seen as the sum of the contributions coming from the agonist and antagonist side, yielding:

$$
\triangle q=\frac{\partial \hat{q}}{\partial \hat{\tau}_{\vartheta}} \hat{\tau}_{f}\left(\hat{\tau}_{\vartheta}\right)+\frac{\partial \hat{q}}{\partial \hat{\tau}_{\vartheta^{a}}} \hat{\tau}_{f}^{a}\left(\hat{\tau}_{\vartheta}\right)
$$

From the expression of $\partial \alpha / \partial \tau$ given in Eq. 22 we have:

$$
\frac{\partial \hat{q}}{\partial \hat{\tau}_{\vartheta}}=\frac{U_{2}^{\prime \prime}}{U_{1}^{\prime \prime}+U_{2}^{\prime \prime}} \frac{\partial \hat{q}}{\partial \hat{\tau_{q}}}
$$

and

$$
\frac{\partial \hat{q}}{\partial \hat{\tau}_{\vartheta^{a}}}=\frac{U_{3}^{\prime \prime}}{U_{3}^{\prime \prime}+U_{4}^{\prime \prime}} \frac{\partial \hat{q}}{\partial \hat{\tau}_{q}}
$$

thus substituting those quantities in Eq. (3) yields:

$$
\begin{aligned}
\triangle q & =\frac{U_{2}^{\prime \prime}}{U_{1}^{\prime \prime}+U_{2}^{\prime \prime}} \frac{\partial \hat{q}}{\partial \hat{\tau}_{q}} \hat{\tau}_{f}\left(\hat{\tau}_{\vartheta}\right)+\frac{U_{3}^{\prime \prime}}{U_{3}^{\prime \prime}+U_{4}^{\prime \prime}} \frac{\partial \hat{q}}{\partial \hat{\tau}_{q}} \hat{\tau}_{f}^{a}\left(\hat{\tau}_{\vartheta^{a}}\right) \\
& =\frac{\partial \hat{q}}{\partial \hat{\tau_{q}}}\left(\frac{U_{2}^{\prime \prime}}{U_{1}^{\prime \prime}+U_{2}^{\prime \prime}} \hat{\tau}_{f}\left(\hat{\tau}_{\vartheta}\right)+\frac{U_{3}^{\prime \prime}}{U_{3}^{\prime \prime}+U_{4}^{\prime \prime}} \hat{\tau}_{f}^{a}\left(\hat{\tau}_{\vartheta^{a}}\right)\right) .
\end{aligned}
$$

Eq. 4 represents the quantity we were looking for: it analytically defines the global actuator dead-band as a function of the internal system torques (and thus the co-contraction level). In particular we have been able to define how the static friction on the gearbox is reflected on the output joint.

To improve the performance of our system we now formulate a differential condition on the spring potential energies to guarantee that co-contraction reduces the effect of gearbox friction on the joint equilibrium position. To make the analysis as general as possible, the conditions are expressed by assuming a generic functional dependence between stiction $\tau_{f}$ and applied torque $\tau$. Similarly the non-linear spring potential energies are kept unspecified.

We want to study how Eq. (4) varies with variations of $\hat{\tau}_{\vartheta}$ and $\hat{\tau}_{\vartheta^{a}}$. The first term is the joint sensitivity, i.e. the inverse of the joint stiffness, and it monotonically decreases given a proper control action, for example by a co-contraction. We are then left to study the remaining quantities inside the brackets. Because of the symmetry of the actuator we can focus our analysis on the antagonist term only $]^{4}$. By taking its derivative

\footnotetext{
${ }^{4} \mathrm{We}$ choose the actuator antagonist side because in this case torque and angular position of the capstan are positive for co-contraction as defined in Fig. 3 Furthermore for the sake of notation simplicity we drop the superscript "a".
}

with respect to $\hat{\tau}_{\vartheta}$ we get:

$$
\begin{aligned}
& \frac{\partial}{\partial \hat{\tau}_{\vartheta}}\left(\frac{U_{3}^{\prime \prime}}{U_{3}^{\prime \prime}+U_{4}^{\prime \prime}} \hat{\tau}_{f}\left(\hat{\tau}_{\vartheta}\right)\right)= \\
& =\frac{U_{3}^{\prime \prime} U_{4}^{\prime \prime \prime}\left(\frac{\partial \hat{\vartheta}}{\partial \hat{\tau}_{\vartheta}}+\frac{\partial \hat{q}}{\partial \hat{\tau}_{\vartheta}}\right)-U_{3}^{\prime \prime \prime} U_{4}^{\prime \prime} \frac{\partial \hat{\vartheta}}{\partial \hat{\tau}_{\vartheta}}}{\left(U_{3}^{\prime \prime}+U_{4}^{\prime \prime}\right)^{2}} \hat{\tau}_{f} \\
& +\frac{U_{3}^{\prime \prime}}{U_{3}^{\prime \prime}+U_{4}^{\prime \prime}} \hat{\tau}_{f}^{\prime}
\end{aligned}
$$

where for the sake of notation simplicity we drop the dependency of $\hat{\tau}_{f}$ from $\hat{\tau}_{\vartheta}$ and denote $\frac{\partial \hat{\tau}_{f}}{\partial \hat{\tau}_{\vartheta}}\left(\hat{\tau}_{\vartheta}\right)$ as $\hat{\tau}_{f}^{\prime}$. This simple expression can be exploited to define the condition in order for the dead-band $\Delta q$ to decrease:

$$
\frac{\partial}{\partial \hat{\tau}_{\vartheta}}\left(\frac{U_{3}^{\prime \prime}}{U_{3}^{\prime \prime}+U_{4}^{\prime \prime}} \hat{\tau}_{f}\right)<0 .
$$

Equation (6) has a great importance because it represents the criterion to which the new non-linear spring must conform. Furthermore substituting the expression of $U_{3}$ and $U_{4}$ of the first prototype of the actuator it has been possible to confirm that Eq. 6 is not satisfied and thus to explain why the current choice of springs and gearboxes originated a deadband monotonically increasing with co-contraction.

\section{DERIVATION OF SPRINGS POTENTIAL ENERGIES}

The problem of finding a solution for our differential inequality (6) can be tackled from different perspective and using different methods. One option is, for example, to solve an optimization problem, while another option is to solve the problem analytically for easy potential energies (e.g., polynomial up to the 3rd order). In both cases anyway we wouldn't obtain adequate results, as a consequence of the fact that the optimization involves a large number of parameters and that we already impose the solution structure (i.e., 3rd order polynomial). Among all the possibilities, we decide to adopt a different method. We consider the dead-band condition as a single functional of $\tau_{\vartheta}$ relating the potential energy of both springs to each other. With this approach we solve the differential equation and make explicit the relation between serial and parallel elastic elements. Eventually, to find the spring characteristic equation, we rewrite the equations as functions of capstan angular position $(\vartheta)$ instead of capstan torque.

Starting from Eq. 6 we construct an augmented form introducing a non-negative slack variable to transform the differential inequality into an equality. We choose the slack variable to be quadratic to easily guarantee its positivity:

$$
\lambda=\left(\frac{u}{\tau_{\vartheta}}\right)^{2}
$$

where $u$ represents the dead-band reduction rate. Furthermore we define the functional $T\left(\tau_{\vartheta}\right)$ as follows:

$$
T\left(\tau_{\vartheta}\right)=\frac{U_{3}^{\prime \prime}\left(\tau_{\vartheta}\right)}{U_{4}^{\prime \prime}\left(\tau_{\vartheta}\right)+U_{3}^{\prime \prime}\left(\tau_{\vartheta}\right)}
$$

where it is important to notice that $U_{3}^{\prime \prime}$ and $U_{4}^{\prime \prime}$ are derivative of the potential energy with respect to $\vartheta$ but written as function of 
$\tau_{2}^{5}$ By using (7) and 8 we can rewrite Eq. 6 in the following simplified form:

$$
\frac{\partial}{\partial \tau_{\vartheta}}\left(T\left(\tau_{\vartheta}\right) \tau_{f}\right)+\lambda=0
$$

We can compute its derivative obtaining the following differential equation in $T\left(\tau_{\vartheta}\right)$ :

$$
\mu \tau_{\vartheta} T^{\prime}\left(\tau_{\vartheta}\right)+\mu T\left(\tau_{\vartheta}\right)+\frac{u^{2}}{\tau_{\vartheta}^{2}}=0
$$

and its solution:

$$
T\left(\tau_{\vartheta}\right)=\frac{u^{2}}{\mu \tau_{\vartheta}^{2}}+\frac{C}{\tau_{\vartheta}},
$$

where $C$ represents the constant of integration and $\mu$ represent the static friction coefficient ${ }^{6}$ Exploiting the definition of $T\left(\tau_{\vartheta}\right)$ it is possible to express $U_{4}^{\prime \prime}$ as function of $U_{3}^{\prime \prime}$ :

$$
U_{4}^{\prime \prime}=\frac{U_{3}^{\prime \prime}-T\left(\tau_{\vartheta}\right) U_{3}^{\prime \prime}}{T\left(\tau_{\vartheta}\right)}=\left(\frac{1}{T\left(\tau_{\vartheta}\right)}-1\right) U_{3}^{\prime \prime} .
$$

Eq. 12 is the mathematical counterpart of the concept we mentioned before, i.e. to write the dead-band condition as a functional of $\tau_{\vartheta}$ and to relate the potential energy of the springs to each other. This equation will be used to derive the parallel spring potential energy given the series one.

The last step to complete the analytical model requires the introduction of a new equation relating the potential energies and the capstan angular position. This can be done by having a look again at the sensitivity matrix in Eq. 2. In particular we focus our analysis on the term $\frac{\partial \hat{\vartheta}^{a}}{\partial \hat{\tau}_{\vartheta a}^{a}}$, which represents the sensitivity of the internal capstan position with respect to the internal actuation torque. This term, simplifying the notation, is:

$$
\frac{\partial \vartheta}{\partial \tau_{\vartheta}}=\frac{U_{1}^{\prime \prime} U_{2}^{\prime \prime}+U_{1}^{\prime \prime} U_{3}^{\prime \prime}+U_{2}^{\prime \prime} U_{3}^{\prime \prime}}{U_{1}^{\prime \prime} U_{2}^{\prime \prime} U_{3}^{\prime \prime}+U_{1}^{\prime \prime} U_{2}^{\prime \prime} U_{4}^{\prime \prime}+U_{1}^{\prime \prime} U_{3}^{\prime \prime} U_{4}^{\prime \prime}+U_{2}^{\prime \prime} U_{3}^{\prime \prime} U_{4}^{\prime \prime}} .
$$

To simplify this equation, we can consider that adopting the co-contraction strategy implies that the agonist and antagonist capstan torques are equal in magnitude, i.e., $\left|\tau_{\vartheta}\right|=\left|\tau_{\vartheta^{a}}\right|$. As a consequence, the net torque acting on the output joint is 0 because each actuator balances the other. Conceptually, this particular situation, from the antagonist motor side, is experienced as if the cable connecting the series elastic element to the joint were attached to an infinitely stiff spring. Thanks to this observation we can simplify Equation 13 considering the

\footnotetext{
${ }^{5}$ This can be done if we consider that the angular position of the capstan is a function of the applied torque: $\vartheta\left(\tau_{\vartheta}\right)$. In particular if the agonist and antagonist torques are equal in magnitude the capstan position can be computed inverting the following equilibrium equation:
}

$$
\tau_{\vartheta}=U_{P E}^{\prime}(\vartheta r) r+U_{S E}^{\prime}(\vartheta r) r
$$

\footnotetext{
${ }^{6}$ As shown in Section $\mathrm{VI}$ the stiction torque $\tau_{f}$ is a linear function of the capstan torque: $\tau_{f}=\mu \tau \vartheta$
}

agonist elastic elements, whose potential energies are labeled $U_{1}$ and $U_{2}$, as if they have infinite stiffness, thus obtaining:

$$
\frac{\partial \vartheta}{\partial \tau_{\vartheta}}=\frac{1}{U_{3}^{\prime \prime}+U_{4}^{\prime \prime}}
$$

Substituting Eq. 11 and Eq. 12 into Eq. 14 we obtain a simple equation relating the antagonist serial spring and the antagonist capstan sensitivity:

$$
\frac{\partial \vartheta}{\partial \tau_{\vartheta}}=\frac{u^{2}+\mu \tau_{\vartheta} C}{\mu \tau_{\vartheta}^{2} U_{3}^{\prime \prime}}
$$

Now, choosing an appropriate $U_{3}^{\prime \prime}{ }^{7}$ and integrating Eq. 15 , we obtain the relation between the capstan angular position and the applied torque:

$$
\begin{aligned}
U_{3}^{\prime \prime} & =k \tau_{\vartheta}^{2} \\
\vartheta\left(\tau_{\vartheta}\right) & =\int \frac{u^{2}+\mu \tau_{\vartheta} C}{\mu \tau_{\vartheta}^{2} k \tau_{\vartheta}^{2}} d \tau_{\vartheta}=\frac{2 u^{2}+3 \mu \tau_{\vartheta} C}{6 k \mu \tau_{\vartheta}^{3}}
\end{aligned}
$$

If we solve Eq. 17 for $\tau_{\vartheta}(\vartheta)$ we obtain three different solutions: two are complex conjugates and one is real. We consider only the real one:

$$
\tau_{\vartheta}=\frac{\sqrt[3]{6} k \vartheta C \mu^{2}-\sqrt[3]{\gamma^{2}}}{\sqrt[3]{6 \gamma} k \vartheta \mu}
$$

where

$$
\gamma=\sqrt{6 k^{3} \mu^{4} \vartheta^{3}\left(C^{3} \mu^{2}+6 k \vartheta u^{4}\right)}+6 k^{2} \mu^{2} \vartheta^{2} u^{2} .
$$

Substituting Eq. 18 into Eq. 16 and Eq. 12 we obtain the functionals we were looking for.

As a simple test to prove the validity of our approach we substitute the second derivative with respect to $\vartheta$ of the new potential energies, written as a function of $\tau_{\vartheta}$, inside the deadband condition (6). What we obtain is an always negative derivative with slope given by the slack variable (7) $\lambda$ :

$$
\frac{\partial}{\partial \hat{\tau}_{\vartheta}}\left(\frac{U_{3}^{\prime \prime}}{U_{4}^{\prime \prime}+U_{3}^{\prime \prime}} \hat{\tau}_{f}\right)=-\frac{u^{2}}{\tau_{\vartheta}^{2}}
$$

The main result of this section is represented by the analytical expression of the two potential energies. The two solutions are both characterized by the presence of a vertical asymptote and have three free variables, i.e. $k, u$ and $C$, which can be chosen to tune the shape of the two curves for a given actuator.

\section{EXPERIMENTAL TESTS}

In this section, through experimental test, we validate the analytical solutions previously derived. In particular, tests are conducted in two different phases: first we measure the dead-band amplitude of the current actuator and we estimate the gear static friction coefficient $\mu$. Subsequently, through numerical optimization, we adapt the analytical expressions of the non-linear spring potential energies to our current motor subsystems and we test the new springs.

\footnotetext{
${ }^{7}$ A potential function which guarantees the positivity and the monotonically increasing property assumed in Section IV written as a function of $\tau_{\vartheta}$.
} 


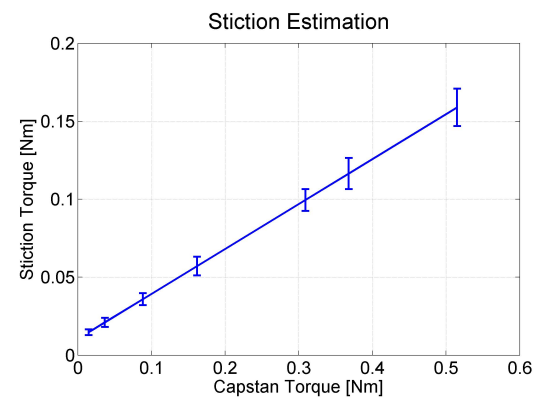

Fig. 8. Experimental tests on the motor subsystem to estimate the static gear friction. This plot reveals the linear behavior for which the corresponding regression coefficient $\mu$ has been estimated to 0.28 . The plot also shows the error bars for the measured data.

\begin{tabular}{cc}
\hline $\begin{array}{l}\text { Torque } \\
{[\mathrm{Nm}]}\end{array}$ & DB [deg] \\
\hline 0.12 & 3.5 \\
\hline 0.25 & 27.9 \\
\hline 0.50 & 69.9 \\
\hline 1.00 & 85.5 \\
\hline
\end{tabular}

TABLE I

THE TABLE SHOWS HOW THE DEAD-BAND (NAMED “DB") INCREASES WITH CO-CONTRACTION.

\section{A. Current actuator performances}

Exploiting the output joint position sensor [26], the amplitude of the actuator dead-band has been measured for a given set of equal internal actuation torques. Results have been recapped in Table 1 It emerges clearly that by increasing the level of co-contraction the amplitude of the dead-band increases.

The static friction coefficient $\mu$ has been evaluated by carrying out the following experiment for each motor subsystem. We applied to the motor a known constant torque and we wound a steel cable around the capstan. We applied to it a known and measurable force and we started to progressively increase it until the capstan started to rotate. In this way we obtained the real torque at the load, $\tau$. Knowing the gear ratio $\rho$ it has been then possible to calculate the expected $\tilde{\tau}$ and to estimate the friction component $\tau_{f}$. The results of the tests, shown in Fig. 8 , reveal the linear behavior of the friction, for which it has been possible to estimate the coefficient $\mu=0.28$.

\section{B. New spring design and test}

To design a suitable set of non-linear springs for the current actuator we can shape the analytical solutions through the three variables $k, u$ and $C$. Given the complexity of the formulas, that makes impossible to mathematically explore the space of all feasible values, we decide to tackle the problem by using numerical tools through a multi-variable optimization. To define the feasible domain, we consider two kinds of constraints: algebraic and design constraints. Algebraic constraints can be derived from the expression of $U_{3}^{\prime \prime}$ and $U_{4}^{\prime \prime}$, as seen in the previous section. In particular, to guarantee the physical consistency of our mathematical analysis we need to guarantee that:

$$
6 k^{3} \mu^{4} \vartheta^{3}\left(C^{3} \mu^{2}+6 k \vartheta u^{4}\right)>0
$$

The variable $u$ represents the reduction rate of the dead-band: the higher the value of $u$, the faster the dead-band decreases during co-contraction. This implies that we need to find a good compromise that guarantees the validity of our analytical model and maximizes the effectiveness of the new springs. Regarding design constraints, we have some restrictions such as limited space and motors' peak torque of about $1 \mathrm{Nm}$. Furthermore we need to consider in our optimization problem the possibility to cover a wide range of stiffness and maximize the operational range of the output joint.

The optimization has been split into two steps: the first part checks the consistency of the spring energy potentials, while the second step numerically simulates the actuator co-contraction. As presented in Algorithm 1 the first step

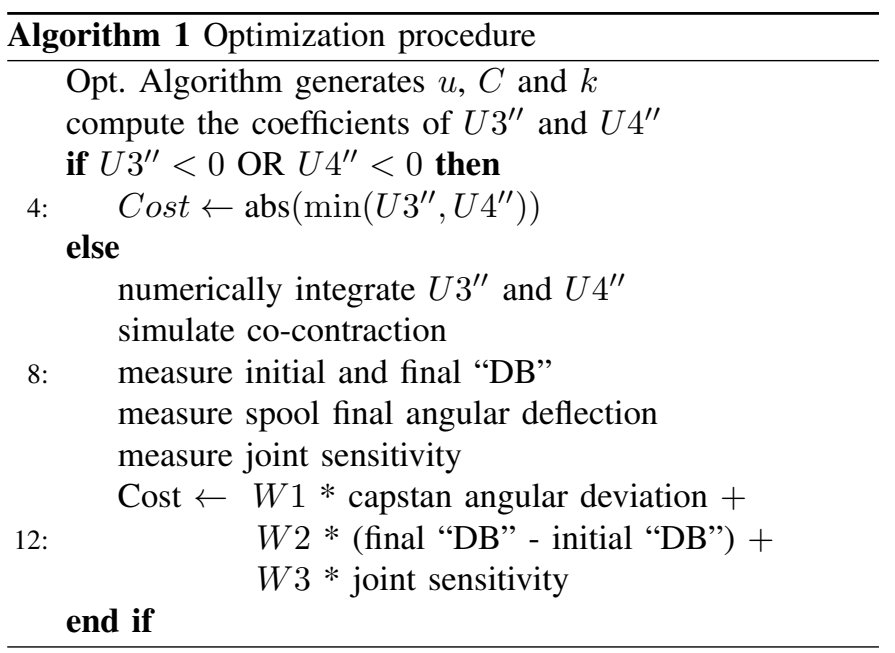

is composed by three lines of the code, which generate a value for the three variables, compute the non-linear spring coefficients $U_{3}^{\prime \prime}$ and $U_{4}^{\prime \prime}$ and test them to verify that there are no negative values. If this is the case, the cost is defined in such a way as to drive the optimization towards positive spring coefficients. The second step of the algorithm is executed only if all the stiffness coefficients are positive. In this case we obtain the spring potential energies by performing a double numerical integration. We then simulate a co-contraction phase increasing the torque from a minimum value up to the motors' peak torque. During the numerical simulation we measure the initial and final dead-band (named "DB"), the final torsion of the capstan and the joint sensitivity. Eventually, the objective function is the weighted sum of the capstan angular deviation from the desired value ${ }^{8}$, the dead-band decrease and the final joint sensitivity 9

Structuring the objective function into two separate subfunctions has the advantage of guiding the algorithm near the feasible set, i.e. in our case avoiding negative stiffness, but it has the drawback of creating a discontinuity which can be lead to a local minimum. To face this issue, and considering also

\footnotetext{
${ }^{8}$ This quantity is essential for the compatibility of the new springs with our former hardware because guarantees that the springs are not either too weak or too stiff.

${ }^{9}$ Decreasing the joint sensitivity has the effect of increasing its stiffness
} 

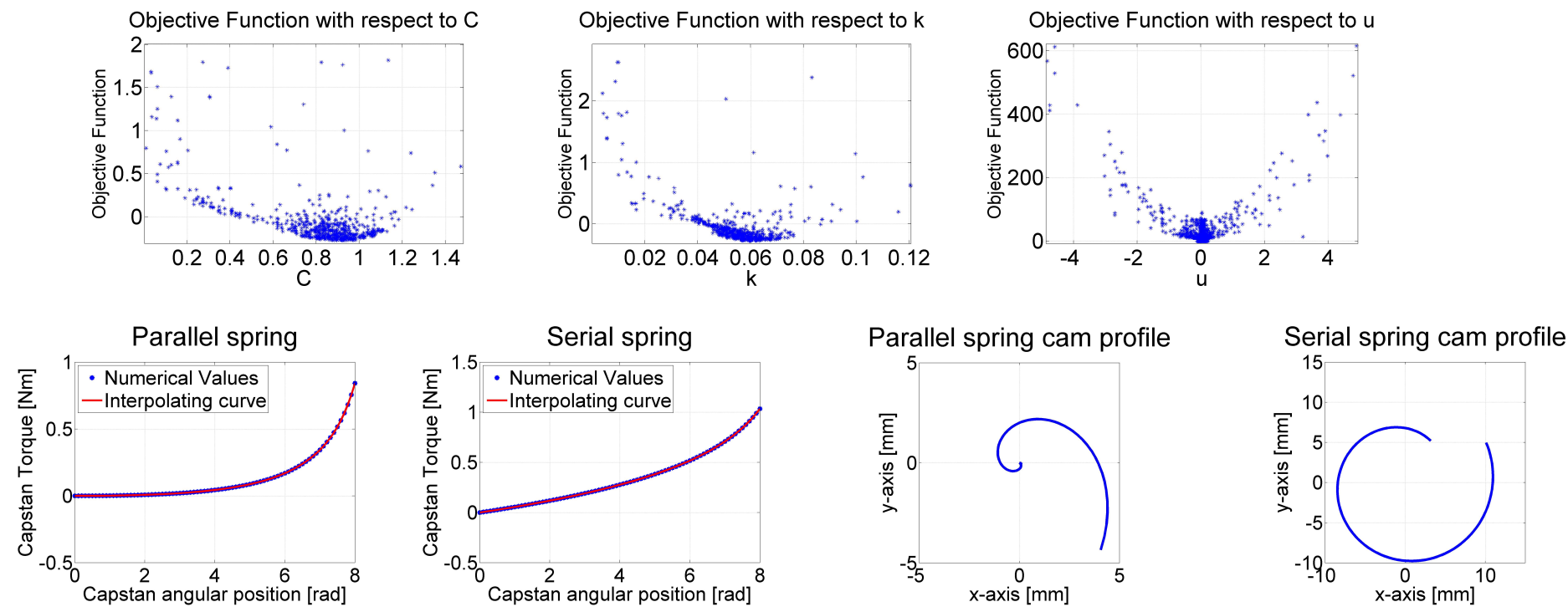

Fig. 9. The plots show the main results of the optimization. In particular, the first three figures represents the behavior of the objective function with respect to the three free variables. In each plot is shown the objective function behavior without keeping constant the other two variables. In the second row are shown the characteristics of the parallel and serial springs together with the relative appearance of the non-circular cams designed to construct the non-linear springs.

that the co-contraction simulation is highly time demanding, we adopt an optimization algorithm based on a multi-process implementation of Differential Evolution [27]. Furthermore we tune the weight $W_{2}$ in order to locate the optimal solution in the region of "negative cost" which can not be reached by the first step. Figure 9 shows the main results of the optimization. In particular, it is interesting to notice how the objective function behaves symmetrically with respect to the free variable $u$ and how the techniques implemented to avoid objective function discontinuities have been effective. We also present the two non-linear spring functions for which vertical asymptotes are around 9 radians, and their respective noncircular cam profiles that have been used to design the new serial and parallel elastic elements.

The last step has been the mechanical realization of the springs and the reassembling of the complete actuator to perform the experimental test. As with the previous configuration we stimulate simultaneously both electric motors in order to produce similar torques at the internal capstans for different levels of co-contraction. The results are shown in Table II which highlights the effectiveness of the novel components in inverting the dead-band mechanical behavior. In particular, given the optimization objective function, the actuator shows a large dead-band for low torques. The last experiment has been carried out increasing the motor current over its nominal value for a short period of time.

\section{CONCLUSIONS}

In this paper we addressed and solved the problem of quantifying analytically the effects and the propagation of internal static friction for a prototype of agonist-antagonist variable stiffness actuator. The novelty and the contribution of our work resides in the possibility of exploiting the elastic elements present in a wide class of agonistic VSA to

\begin{tabular}{cc}
\hline $\begin{array}{l}\text { Torque } \\
{[\mathrm{Nm}]}\end{array}$ & DB $[\mathrm{deg}]$ \\
\hline 0.12 & 104.61 \\
\hline 0.25 & 100.81 \\
\hline 0.5 & 87.73 \\
\hline 1 & 61.62 \\
\hline 1.5 & 49.81 \\
\hline
\end{tabular}

TABLE II

THE TABLE SHOWS THE EFFECTIVENESS OF THE NEW SPRINGS.

passively compensate the undesired side effects of internal friction. Through an analytical approach we have been able to generate a closed-form condition over the spring potential energies to decrease the dead-band for increasing levels of co-contraction. In particular, even if we couldn't achieve the dead-band cancellation, we demonstrate that our mathematical approach can reasonably be exploited to design a novel fullystiction-compensated actuator. The principal constraints that limited the effectiveness of the optimization for our current actuator have been the limited torque provided by the motor subsystems, the very narrow space for designing the new components and still some important, but not predominant, sources of friction in the frame subsystem. We aim in future at designing a new actuator from scratch in order to have a smaller set of constraints allowing full exploitation of all the potentiality of our methodology.

\section{ACKNOWLEDGMENTS}

This paper was supported by the FP7 EU projects CoDyCo (No. 600716 ICT 2011.2.1 Cognitive Systems and Robotics), and Koroibot (No. 611909 ICT-2013.2.1 Cognitive Systems and Robotics). 


\section{REFERENCES}

[1] J. Pratt, B. Krupp, and C. Morse, "Series elastic actuators for high fidelity force control," Industrial Robot Journal, vol. 29, no. 3, pp. 234-241, 2002.

[2] R. Van Ham, T. G. Sugar, B. Vanderborght, K. W.Hollander, and D. Lefeber, "Compliant actuator designs," Robotics \& Automation Magazine, IEEE, pp. 16 81-94, 2009.

[3] B. Vanderborght, R. Van Ham, D. Lefeber, T. G. Sugar, and K. W. Hollander, "Comparison of mechanical design and energy consumption of adaptable, passive-compliant actuators," The International Journal of Robotics Research, vol. 28, no. 1, pp. 90-103, 2009.

[4] C. C. de Wit, H. Olsson, K. Astrom, and P. Lischinsky, "A new model for control of systems with friction," in IEEE Trans. on Automatic Control, vol. 40, 1994, pp. 419-425.

[5] P. Tomei, "Robust adaptive friction compensation for tracking control of robot manipulators." IEEE Trans. Automat. Contr., vol. 45, no. 11, pp. 2164-2169, 2000.

[6] V. Parra-Vega and S. Arimoto, "A passivity based adaptive sliding mode position-force control for robot manipulators," in International Journal of Adaptive Control and Signal Processing, 1996, pp. 365-377.

[7] B. Armstrong, "Dynamics for robot control: Friction modelling and ensuring excitation during parameter identification," in Dissertation, Stanford University, 1988.

[8] L. Fiorio, A. Parmiggiani, B. Berret, G. Sandini, and F. Nori, "pnrvsa: human-like actuator with non-linear springs in agonist-antagonist configuration," 2012.

[9] O. Eiberger, S. Haddadin, M. Weis, A. Albu-Sch äffer, and G. Hirzinger, "On joint design with intrinsic variable compliance: Derivation of the dlr qa-joint," pp. 1687 1694, 2010.

[10] F. Petit, M. Chalon, W. Friedl, M. Grebenstein, A. AlbuSchäffer, and G. Hirzinger, "Bidirectional antagonistic variable stiffness actuation: Analysis, design \& implementation," in ICRA, 2010, pp. 4189-4196.

[11] G. Tonietti, R. Schiavi, and A. Bicchi, "Design and control of a variable stiffness actuator for safe and fast physical human/robot interaction." in ICRA. IEEE, 2005, pp. 526-531.

[12] F. Nori, B. Berret, L. Fiorio, A. Parmiggiani, and G. Sandini, "Control of a single degree of freedom noise rejecting-variable impedance," in Proceedings of the 10th international IFAC symposium on Robot Control (SYROCO2012), 2012.

[13] S. Haddadin, A. Albu-schffer, and G. Hirzinger, "Safety evaluation of physical human-robot interaction via crashtesting," in In Robotics: Science and Systems Conf. (RSS2007, 2007, pp. 217-224.

[14] A. Albu-Schaffer, O. Eiberger, M. Grebenstein, S. Haddadin, C. Ott, T. Wimbock, S. Wolf, and G. Hirzinger, "Soft robotics," Robotics Automation Magazine, IEEE, vol. 15 , no. 3, pp. 20-30, 2008.
[15] N. L. Tagliamonte, F. Sergi, D. Accoto, G. Carpino, and E. Guglielmelli, "Double actuation architectures for rendering variable impedance in compliant robots: A review," Mechatronics, vol. 22, no. 8, pp. 1187 - 1203, 2012.

[16] A. Bicchi and G. Tonietti, "Fast and "soft-arm" tactics [robot arm design]," Robotics Automation Magazine, IEEE, vol. 11, no. 2, pp. 22-33, 2004.

[17] M. Fumagalli, S. Ivaldi, M. Randazzo, L. Natale, G. Metta, G. Sandini, and F. Nori, "Force feedback exploiting tactile and proximal force/torque sensing. Theory and implementation on the humanoid robot iCub," Autonomous Robots, vol. 33, no. 4, pp. 381-398, 2012.

[18] A. Del Prete, F. Nori, G. Metta, and L. Natale, "Control of Contact Forces: the Role of Tactile Feedback for Contact Localization," in Intelligent Robots and Systems (IROS), 2012 IEEE/RSJ International Conference on, 2012.

[19] E. Wieser, P. Mittendorfer, and G. Cheng, "Accelerometer based robotic joint orientation estimation," in $\mathrm{Hu}$ manoids, 2011, pp. 67-74.

[20] E. Burdet, R. Osu, D. W. Franklin, T. E. Milner, and M. Kawato, "The central nervous system stabilizes unstable dynamics by learning optimal impedance." Nature, vol. 414, no. 6862, pp. 446-449, Nov 2001.

[21] B. Berret, G. Sandini, and F. Nori, "Design principles for muscle-like variable impedance actuators with noise rejection property via co-contraction," in Humanoid Robots (Humanoids), 2012 12th IEEE-RAS International Conference on, Nov 2012, pp. 222-227.

[22] A. W. Wiegner and R. L. Watts, "Elastic properties of muscles measured at the elbow in man: I. normal control," J Neurol Neurosurg Psychiatry, 1986.

[23] T. McMahon, Muscle, Reflexes, and Locomotion, P. U. Press, Ed., 1984.

[24] S. Koley and A. Singh, "Trends of active range of motion at three important joints in school-going boys of amritsar," Anthropologist, 2008.

[25] N. Schmit and M. Okada, "Synthesis of a non-circular cable spool to realize a nonlinear rotational spring," in International Conference on Intelligent Robots and Systems (IROS2011). IEEE, 2011, pp. 762-767.

[26] "Aea, strain datasheet," Istituto Italiano di Tecnologia, http://wiki.icub.org/wiki/Other_boards.

[27] M. Buhren, "Multicore differential evolution," Matlab File Exchange website. 